\title{
Addressing the Strengthening the Reporting of Observational Studies in Epidemiology (STROBE) Statement in Archives of Plastic Surgery Reports
}

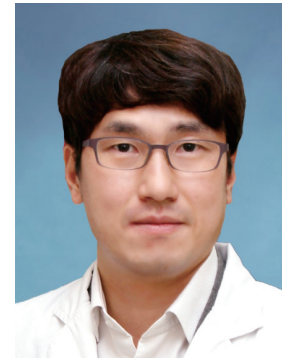

Jang Hyun Lee

Deputy editor, Archives of Plastic Surgery

Department of Plastic and Reconstructive Surgery, Hanyang University College of Medicine, Seoul, Korea

Patient-oriented research is research that makes the patient the subject. While that definition is very broad, it is commonly defined as research conducted with human subjects. Among the topics of patient-oriented research are the usefulness of new technologies, understanding the mechanisms of a disease, therapeutic interventions, and clinical trials. One of the main goals of patient-oriented research is for patients to receive useful benefits from the clinical research findings [1]. Recently, another important concept known as translational research has come to the fore. Commonly expressed as 'from bench-to-bedside', it is the translating of new knowledge, technology, etc., developed in basic science into new processes for effective disease prevention, diagnosis, and treatment. Translational research could be further described as the translation of results obtained from clinical research into actual clinical practice. As translational research always has the patient as its focus, many patients have benefited from the medical developments derived from these numerous patient-oriented research studies [2].

A variety of study designs can be used in patient-oriented research. Among these, randomized controlled trials have been utilized so much that they have come to be considered the gold standard in the field of clinical research in the latter half of the 20th century, and they have had an undeniably profound contribution to the promotion of patient health outcomes up to the present day. However, in randomized controlled trials, individuals may not want to be randomized into a control group, and it may be impossible for randomized selection itself to be ethically acceptable for certain research with an experimental design, such as those on etiology or adverse effects. Particularly within the surgical department, even more than in other medical departments, there are many times when randomized controlled trials are not appropriate due to ethical concerns or feasibility [3].

Another form of research, the observational study, is able to deal with a diverse range of subjects, from new medical discoveries to confirming or disproving previous discoveries, and is more suited to evaluating rare or late side effects related to particular treatments [4]. Because observational studies, including those with study designs such as case-control studies, crosssectional studies, and cohort studies, evaluate treatment efficacy in a non-randomized manner, research can be carried out safely and effectively, especially within surgical departments. Accordingly, as observational studies have recently been found useful in the plastic and reconstructive surgery fields as well, these types of papers have been published in greater numbers in plastic surgical literature [5].

Researchers should carry out scientifically rigorous research and should provide detailed, transparent manuscripts that are easily understandable to the reader. Recommendations have been made for the enhancement of the quality of research reporting, and in 1996, the Consolidated Standards of Reporting Trials was created, which provided support to many medical journals and aided in the improvement of the quality of reports of randomized trials [6]. Afterwards, as previously mentioned, as 
Fig. 1. The Strengthening the Reporting of Observational Studies in Epidemiology (STROBE) statement score

The STROBE statement score for the observational studies published in the Archives of Plastic Surgery.

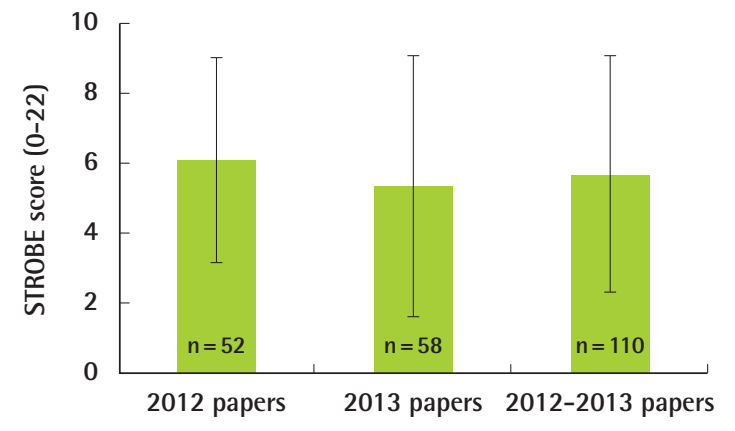

observational studies became more common, a network arose that sought to make recommendations suited to observational studies rather than randomized controlled trials. In 2004, a workshop organized by various concerned parties, including the editorial staff from Annals of Internal Medicine, BMJ, The Lancet, etc., led to the establishment of the Strengthening the Reporting of Observational Studies in Epidemiology (STROBE) Initiative, and in 2007, the STROBE statement was released [6]. The STROBE statement is a checklist of 22 items necessary for good reporting of observational studies. These items address the study design, data collection, variables, statistical methods, and data analysis as appropriate for each section of the article: the title and abstract, introduction, methods, results, and discussion. Eighteen of these items are applicable to all types of study designs, whereas the remaining 4 are designed to be applied specifically to cohort, case-control, or cross-sectional studies.

Archives of Plastic Surgery has set reporting guidelines according to the type of study design of submitted manuscripts in the submission rules and recommends that authors submitting observational studies refer to the STROBE statement. During the two years of 2012 to 2013, among the 143 original articles published in the Archives of Plastic Surgery, 110 (76.9\%) were observational studies. We examined these 110 observational studies to determine how well they complied with the STROBE statement. The results of the assessment, where each of the 22 items was given 1 point for a possible maximum of 22 points, were $5.7 \pm 3.4$ points (Fig. 1). In addition, in our analysis, we noted that many papers stated in the method section that they analyzed the patients retrospectively. In actuality, 60 of 110 papers (54.4\%) took the form of technical notes, applying new surgical techniques to patients or case reports merely illustrating cases, although these papers were original articles. Accordingly, we would like to emphasize our encouragement that the STROBE statement be used in order to improve the reporting quality of observational studies being submitted to Archives of Plastic Surgery.

\section{REFERENCES}

1. Strom BL, Norman S, Margolis DJ. Patient-oriented research: definitions and new paradigms. Am J Med 2000; 109:164-5.

2. Sacristan JA. Patient-centered medicine and patient-oriented research: improving health outcomes for individual patients. BMC Med Inform Decis Mak 2013;13:6.

3. Concato J. Study design and "evidence" in patient-oriented research. Am J Respir Crit Care Med 2013;187:1167-72.

4. Malta M, Cardoso LO, Bastos FI, et al. STROBE initiative: guidelines on reporting observational studies. Rev Saude Publica 2010;44:559-65.

5. Sorensen AA, Wojahn RD, Manske MC, et al. Using the Strengthening the Reporting of Observational Studies in Epidemiology (STROBE) statement to assess reporting of observational trials in hand surgery. J Hand Surg Am 2013; 38:1584-9.e2.

6. von Elm E, Altman DG, Egger M, et al. The Strengthening the Reporting of Observational Studies in Epidemiology (STROBE) statement: guidelines for reporting observational studies. J Clin Epidemiol 2008;61:344-9.

Correspondence: Jang Hyun Lee

Department of Plastic and Reconstructive Surgery, Hanyang University Guri Hospital,

Hanyang University College of Medicine, 153 Gyeongchun-ro, Guri 471-701, Korea

Tel: +82-31-560-2330, Fax: +82-31-560-2338, E-mail: pslee@ hanyang.ac.kr

No potential conflict of interest relevant to this article was reported.

Received: 30 Dec 2013 • Revised: 30 Dec 2013 • Accepted: 30 Dec 2013

pISSN: 2234-6163・ elSSN: 2234-6171

http://dx.doi.org/10.5999/aps.2014.41.1.1 • Arch Plast Surg 2014;41:1-2 